\title{
IOT based Smart Band
}

\author{
Hardik Shekhawat \\ MPSTME, NMIMS \\ Mumbai,India
}

\author{
Tapan Dobhada \\ MPSTME, NMIMS \\ Mumbai,India
}

\author{
Swati Bairagi \\ MPSTME, NMIMS \\ Mumbai, India
}

\author{
Shreyank Sharma \\ MPSTME, NMIMS \\ Mumbai, India
}

\begin{abstract}
Implementing IOT based appliances are bit expensive operation when come to implanting it on home services. Expensive IOT devices are one of the reasons why there is no significant improvement in the IOT home appliances. This paper presents successful implementation of controlling the electrical home appliances by using a smart band. The smart band provides commands via Bluetooth to Android smart phone. An application has been developed for android smart phone specifically transferring the command via internet connectivity to "Ubidots" cloud server and "Raspberry Pi" fetches the command to turn ON or OFF the "GPIO" pins on which relay board is connected. It provides the automation of manual switches for control of electrical appliances.
\end{abstract}

\section{Keywords}

IOT, Hybrid solutions, Home automation, lighting control, nonIOT implementation , smart-band

\section{INTRODUCTION}

The motivation behind this research is to eliminate the energy wastage of electricity and to simplify the IOT for elderly \& physically challenged users. This project will contribute in enhancing the energy saving by reminding the young users on smart phones about the appliances that might have left on while leaving for home and giving them an option to control the devices which can be non-IOT even when outside the home. This research will also focus on smart band developed for users which have difficulty in understanding app functionality, especially elderly and physically challenged people which can simply control the home appliances by using push buttons installed on the band.

\section{RELATED WORK}

In general, intelligent home automation system consists of clusters of sensors, collecting different types of data, regarding the utility consumption at home. Systems with computing capabilities analyse the assimilated data to recognize the activities of inhabitants or events. These can automate the domestic utilizations effectively and also can support the inhabitant by reducing the costs and improving the standard of living. Most of the research activities related to IoT are confined to management of resource constraint devices, and different mechanisms of interconnection. Not only the devices being controlled but home environment can also be continuously monitored desired temperature or monitoring amount of energy consumption. Hence, this will contribute to overall cost reduction and energy saving which is one of the main concerns of today [1].

The rapid advancement of the internet of things (IoT), life is getting easier and simpler in all aspects. At present world, automatic systems are being favored over the manual system. Today's world automation has become an inseparable part of ordinary households and subjects to constant evolution. IoT is a growing network of conventional object-from industry to consumer that can share information and complete jobs while you are involved with other activities. A smart home automation system can help to have a centralized method to control all home appliances. All the devices of this system are connected to Raspberry Pi. The proposed system also provides a facility to control all home appliances locally without the internet via a local network [2].

The existing devices are either bulky or require user intervention. It depends on the user to switch it on and use it. But the unfortunate fact is that whenever a person is attacked, their ability to think logically is lowered making the person quite vulnerable to the judgments made from such state of mind. So the important feature incorporated in the device is its capability to act without any assistance from the user [3].

The cloud-based home automation project is inspired by the necessity to monitor and control the home remotely in an affordable way. Today, technology innovations are being focused more than ever on the Internet of Things (IoT). A world where everything is smart and connected is already work in progress [4].

\section{METHODOLOGY}

To enable home automation the home appliances are connected to solid state 8 port relay which is connected to Raspberry Pi via GPIO connection through connectors. The Raspberry pi is a powerful microcontroller; it is just like a miniature computer and consists of GPIO pins which can be coded to turn HIGH or LOW by writing a program in Python.

The raspberry pi will connect to internet via Wi-Fi module which is inbuilt on the device and establish its connection with desired Android device of the user through cloud server. The Android smartphone consists of an application specifically build for the purpose. The Bluetooth of the device will connect to $\mathrm{HC05}$ Bluetooth module which is in turn connected to Arduino lillypad. It is also a microcontroller specially designed for wearable purposes. This device will consist of push button which will signal the android app that a button is pressed and send the set of instruction to raspberry pi to turn ON or OFF the specific GPIO pins. The GPIO pins will turn on or off the relay port which will turn on or off the devices connected to the port

The system combined can work to automate the home appliances with ease. The involvement of cloud is very crucial for this project as it gives the users flexibility to operate the smart band at any place, any time and without any Hassel. The mobile phone and raspberry requires an active internet connection to maintain the link and successful operation of the project. The project can eliminate the requirement of manual switches and currently available electrical appliances of IOT. The project components have a very simplistic approach and the module can be installed at any place in the home with area near the switch board is preferable. The architecture is represented below . 


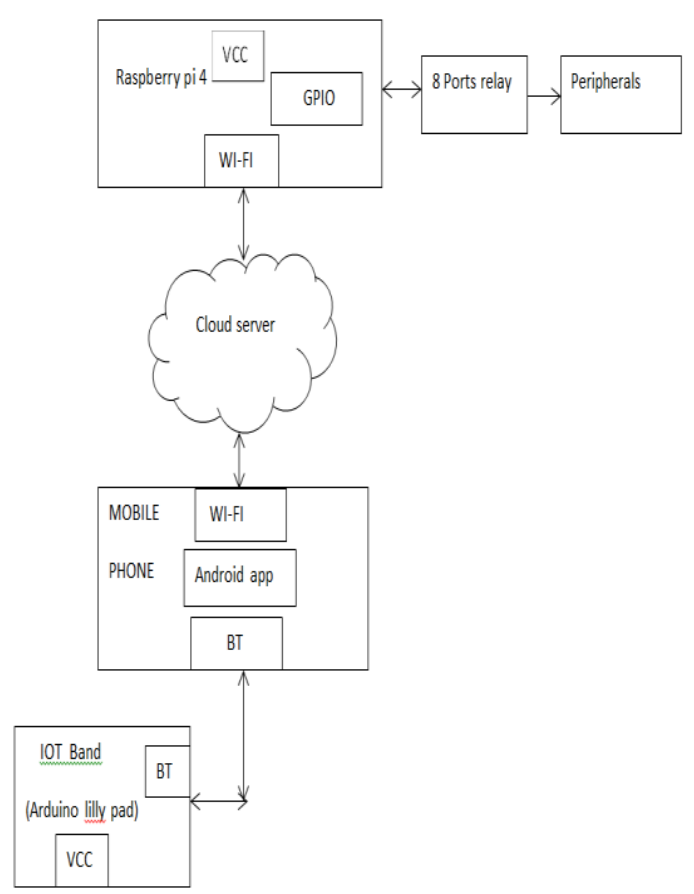

Fig. 1 Architecture of IOT based Smart Band

\section{IMPLEMENTATION}

The push buttons are successfully integrated on the Arduino Lillypad with HC-05 Bluetooth module. When the button is pressed the Arduino Lillypad prompt a feedback light, hence providing a visual signal to the users that a button is currently pressed on the device it is also shown in the figure 2 below. The Arduino lillypad can be connected to $5 \mathrm{~V}$ output lithium ion battery for its power source. Since, Arduino lillypad can be coded to perform the specific function, it can easily configured and customized as per the user requirement.

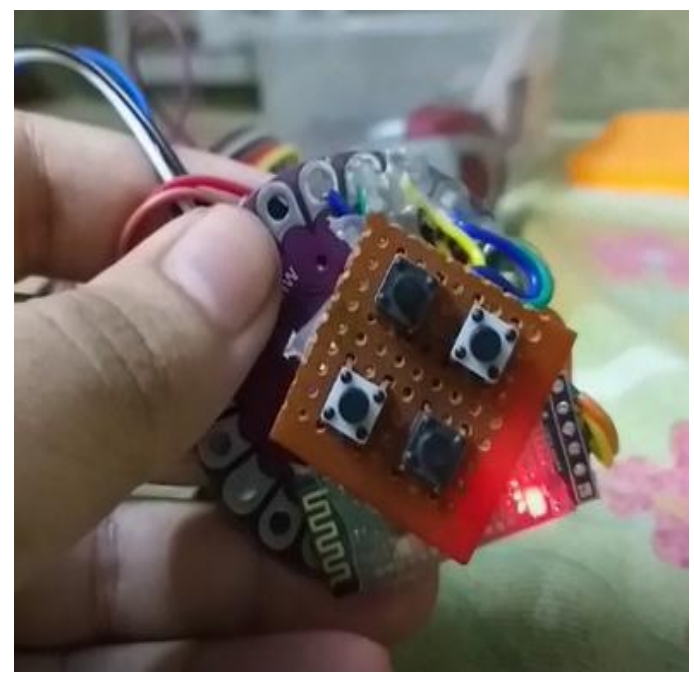

Fig.2 Smart Band prototype

The Hc-05 module connects to android smartphone via integrated Bluetooth chip and form a SSP connection for communication. Once the pairing is complete, an Android application needs to be accessed which is specifically build for this project as shown in figure 3 below. Hence, when the button is pressed the application also indicates about which button is pressed and forwards it to the cloud services.

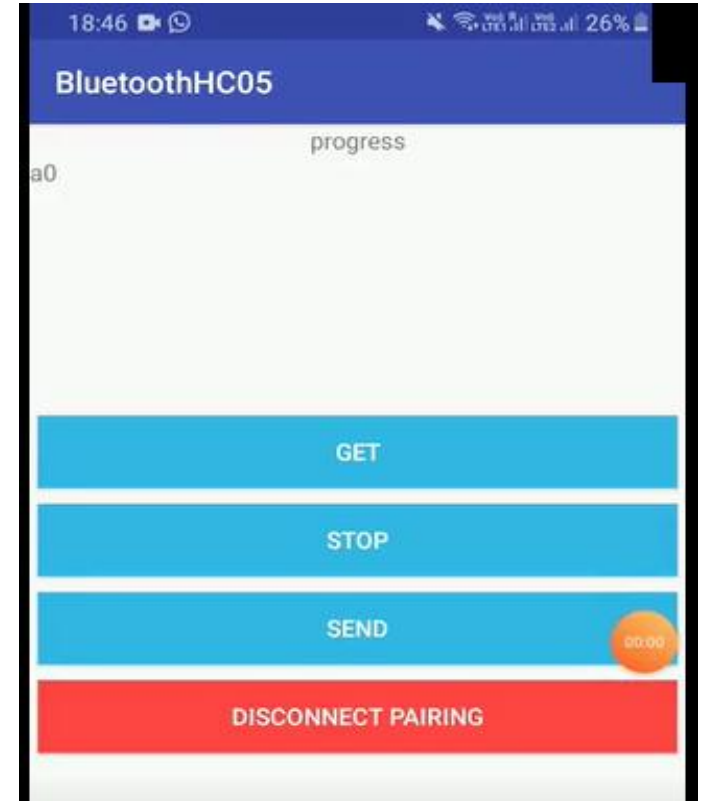

Fig.3 Android Application displaying currently pressed button

Once the android app. Transfers the data to cloud platform the pre-configured cloud API stores the information about the key which is currently pressed into its specifically configured window as shown in figure4 below. The information is continuously fetched on to the cloud from the android application to reduce delay.

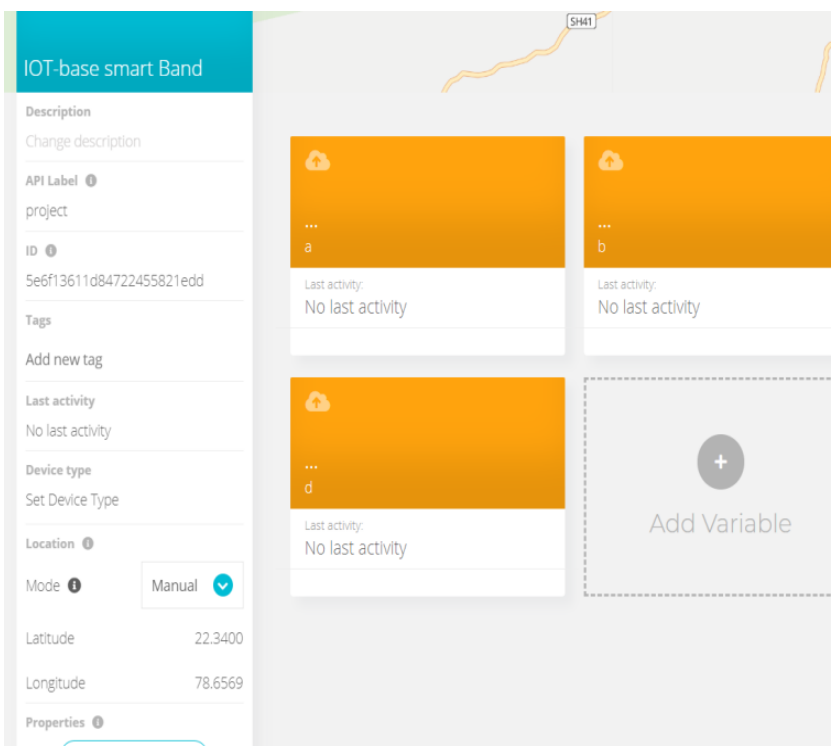

Fig. 4 IOT cloud service configurations for push buttons

The cloud generates a static unique ID for variables as well as the project which is programmed in both android application as well the python code running on raspberry pi. Once the information of changes in key is stored on cloud the python code running on raspberry pi fetches the code after every 5 seconds and turn on or off the GPIO pin of raspberry pi which in turn perform the switching mechanism of 8 port SSD relay as shown in Figure. 5 below. 


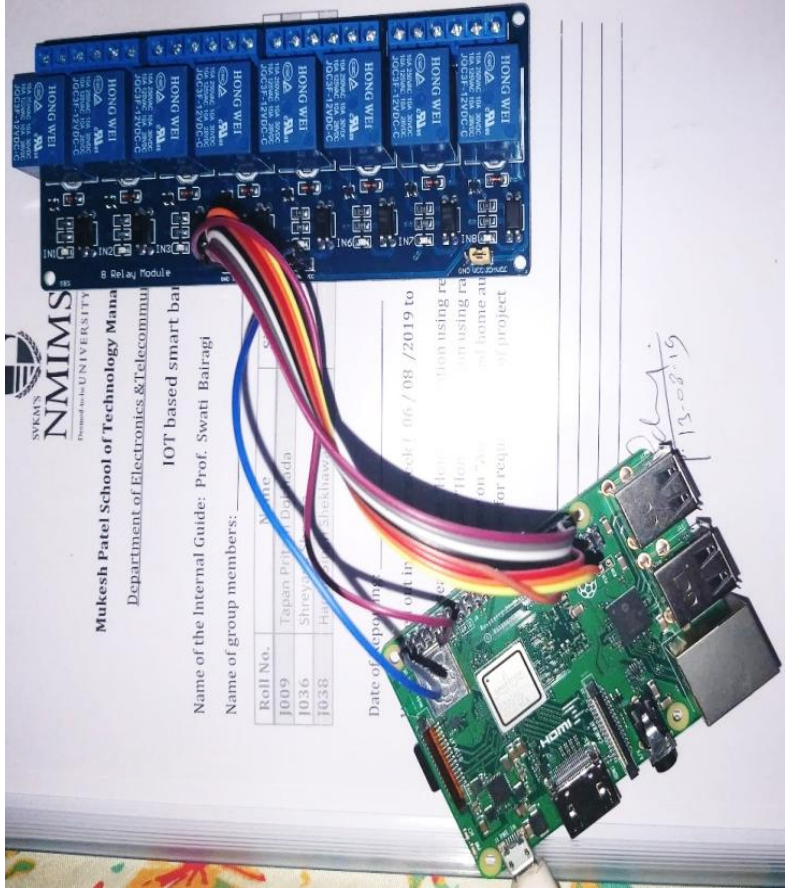

Fig. 5 Raspberry Pi and relay board connection

\section{FLOW CHART}

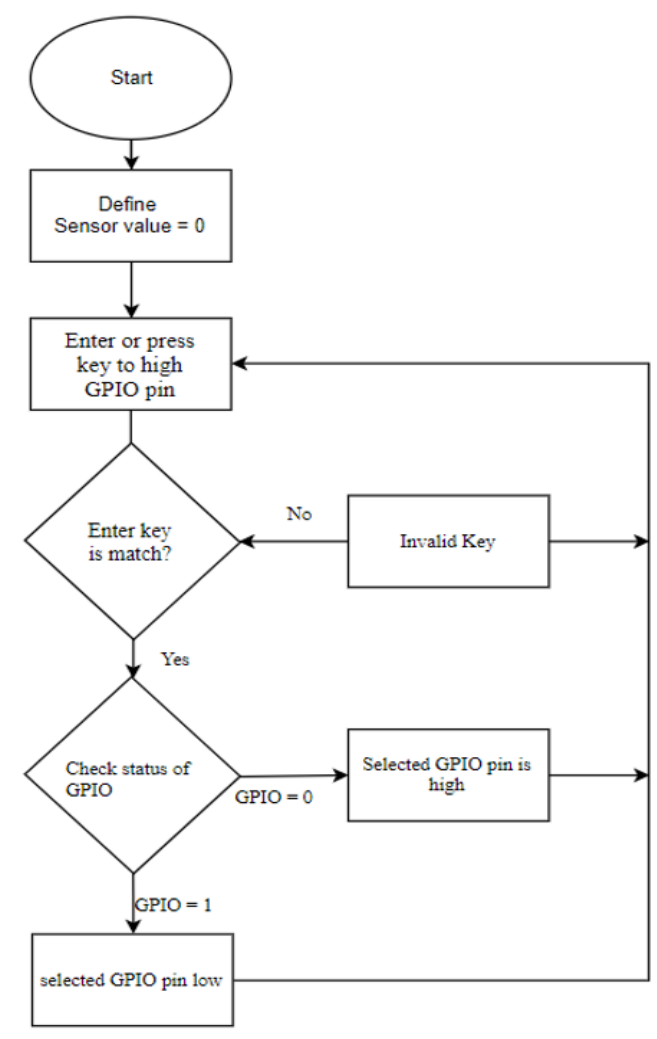

Fig. 6 Relay board implementation flow chart

\section{RESULT}

The result of the project was successful and the initial aim and implementation is operating successfully as it was thought during ideation stage. The functioning of the project is currently at basic level, which means that it can be implemented in the home and can substitute the manual switches.
Manual switches can be completely or partially substituted because the controlling of these analog devices can be done wirelessly at any part of the world which means when the smart band and raspberry pi are connected to the internet, the relay board can be controlled by the band anywhere irrespective of the location of the user. it has been observed that the raspberry pi and cellular device must have an active internet connection at all of the time. Since, the whole working is dependent on internet; a minute fault in internet connectivity may break the chain and can cause the communication loss between cloud and raspberry pi or between cloud and mobile phone.

\section{CONCLUSION}

IOT is the technology that enables the devices to communicate between each other through unique ID. The unique ID helps them to identify the devices to communicate with and further precedes the instructions. The current IOT devices are specific to its own appliances and android application rather than generalized appliances which creates a gap for users in middle or lower class segment for application of these devices at their home. The project is an innovative way to implement IOT technology. It deals with targeting the manual switches installed at houses, offices \& industries and automates the process by providing the control on users' phone or on their hands by using a smart band.

The results indicate that the devices works perfectly for extended period of time without any technical issues and can easily switch the GPIO pins which eventually switch the relay ports. The cloud servers actively monitor the individual push button for any recent changes or if the any button is pressed recently and transmits the information to the pi. The transmission is secured as each button has its own unique ID which is encrypted and cannot be tapped easily. Any device can be connected on this network for operation without much or any change in the configuration of the system.

\section{REFERENCES}

[1] N.Selvi, M.S.Balamurugan, "Design and Control of Internet of Things Enabled Wireless Sensor Network", International Journal of Engineering Sciences \& Research Technology [3722-3726].

[2] Bepery, Chinmay \& Baral, Sudipto \& Khashkel, Animesh \& Hossain, Farhad. (2019). Advanced Home Automation System Using Raspberry-Pi and Arduino. International Journal of Computer Science and Engineering (IJCSE) Vol. 8, Issue 2, Feb - Mar 2019; 1-10.

[3] P N, Vivek \& Sundara Murthy, Sushma \& R C, Suhas \& S, Reshma \& Ramakrishna, Raksha \& J, Roopa. (2013) Design and Implementation of Smart Wrist band for Safety Measures in Emergency. 10.13140/RG.2.2.17943.78241. Proceedings of National Conference on Wireless Communication, Signal Processing, Embedded SystemsWISE 2013.

[4] Katta Nagendra Babu , Khagga Vijaya Lakshmi , K ondaveeti Sai Bhargavi , Akula Ravi , Katta Sasidhar," IOT Based Home Automation System Using Raspberry Pi with Web Server", International Journal of Innovative Research in Science, Engineering and Technology Vol. 7, Issue 3, March 2018.

[5] Miloš Milošević, Nenad Četić, Jelena Kovačević, Tihomir Anđelić, "Lighting Control Using Raspberry Pi and Oblo Living Home Automation System", SERBIAN JOURNAL OF ELECTRICAL ENGINEERING Vol. 16, No. 1, February 2019, 45-54 
[6] Wankhade, Ravi, Shashank Karhade, Pratik Mohite, Kanchan Dhole, Akash Ganvir, Bharti Khedkar and Sharayu S. Sangekar. "Home Automation System Based on IOT using Cellular Devices.", DOI:10.32628/IJSRST196180 Corpus ID: 88499172 (2019).

[7] Modi, Divyang. "A Review of Home Automation Systems.” (2019).

[8] Swapnil Thakare, Sharad Yadav, Amar Waghade, "IoT and AI bases Home Automation System", International Journal of Research in Engineering, Science and
Management Volume-2, Issue-2, February-2019.

[9] Kalyan Kumar Jena , Sourav Kumar Bhoi , Pabitra Kumar Maharana , Prabhas Ranjan Das , and Prabin Kumar Senapati ," A Smart and Secure Home Automation System Using IoT", Universal Review, Volume VIII, Issue III, MARCH/2019 ISSN NO : 2277-2723 Page No: 125.

[10] Park, Jintae \& Hwang, Hyun-Seo \& Moon, Il-Young. (2014). Study of Wearable Smart Band for a User Motion Recognition System. International Journal of Smart Home. 8. 33-44. 10.14257/ijsh.2014.8.5.04. 